
\section{$\zeta=$}

${ }^{1}$ Respiratory Medicine Department, Kings Mill Hospital, Sutton-in-Ashfield, UK

${ }^{2}$ Academic Unit of Radiology, University of Sheffield, Royal Hallamshire Hospital, Sheffield, UK

${ }^{3}$ Sheffield Pulmonary Vascular Disease Unit, Royal Hallamshire Hospital, Sheffield Teaching Hospitals NHS Foundation Trust, Sheffield, UK

\section{Correspondence to}

Dr Amanda T Goodwin Respiratory Medicine Department, Kings Mill Hospital, Sutton-in-Ashfield NG17 4JL, UK:

Amanda.Goodwin@

nottingham.ac.uk

Received 27 July 2015

Revised 6 January 2016

Accepted 20 January 2016

Published Online First

12 February 2016

\title{
Breathlessness in an ex-miner: an unusual consideration
}

\author{
Amanda T Goodwin, ${ }^{1}$ Jillian Wall, ${ }_{1}$ Smitha Rajaram, ${ }^{2}$ Khaled Amsha, ${ }^{1}$ David G Kiely ${ }^{3}$
}

\section{CLINICAL PRESENTATION}

A 73 -year-old retired miner, with 36 years 'at the coal face', presented with a 5 -year history of exertional dyspnoea on climbing steep inclines. His medical history was limited to quiescent chronic lymphocytic lymphoma, for which he required no chemotherapy or radiotherapy and had annual haematology follow-up. He was a smoker, with a 15 pack-year history, and took no regular medications.

The patient had a widely split second heart sound, with accentuation of the pulmonary component, and bilateral intercostal bruits. Echocardiography demonstrated normal left sided chambers, moderate mitral and tricuspid regurgitation, and a raised estimated systolic pulmonary artery pressure of $80 \mathrm{~mm} \mathrm{Hg}$. Pulmonary function tests showed an obstructive pattern $\left(\mathrm{FEV}_{1} 1.91 \mathrm{~L}\right.$ (70\% predicted), FVC $3.62 \mathrm{~L}$ (101\%), $\mathrm{FEV}_{1} / \mathrm{FVC}$ ratio $53 \%$ ), with a mildly reduced diffusion capacity (transfer coefficient (KCO) $0.86 \mathrm{mmol} / \mathrm{min} / \mathrm{KPa} / \mathrm{L}, 70 \%$ predicted). On the incremental shuttle walking test, his oxygen saturations dropped from $97 \%$ to $78 \%$ on walking $500 \mathrm{~m}$. An autoimmune screen was negative.

The chest radiograph showed bilateral large pulmonary opacities in the mid-zones (figure 1A). A CT pulmonary angiogram demonstrated bilateral perihilar soft-tissue masses with irregular margins and enlarged calcified mediastinal lymph nodes, producing an overall appearance consistent with progressive massive fibrosis (PMF). Minor centrilobular emphysematous changes and other lung parenchymal changes consistent with pneumoconiosis were also seen on CT scan (images not shown).
Perfusion scintigraphy and MR pulmonary angiography were subsequently performed (figure $1 \mathrm{~B}, \mathrm{D}$ ).

\section{QUESTION}

What rare complication of PMF does this patient have?

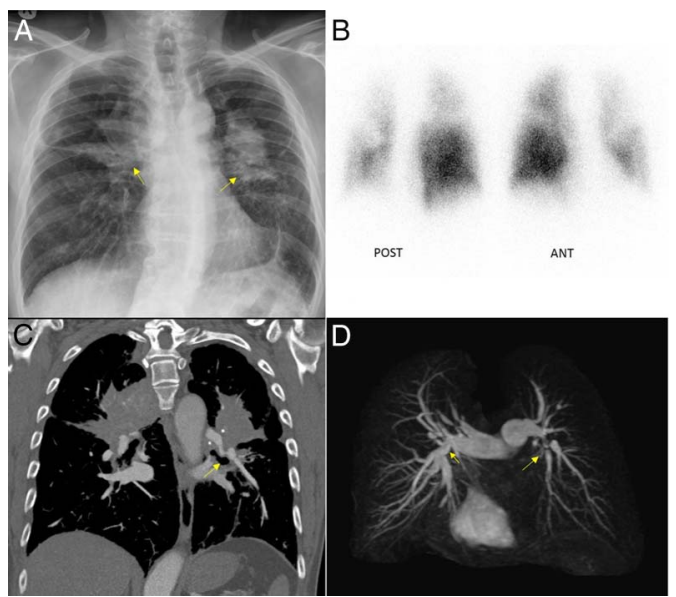

Figure 1 Imaging studies demonstrating extrinsic compression of the pulmonary arteries by the conglomerate masses of progressive massive fibrosis (PMF). (A) Chest radiograph showing large bilateral mass-like conglomerate lesions (arrows). (B) Coronal perfusion scintigraphy image showing large bilateral perfusion defects. (C) Coronal reconstructed CT highlighting narrowing of the left pulmonary artery (arrow) secondary to the large mass-like perihilar lesions of PMF. (D) MR angiographic maximum intensity projection reconstructed images showing significant narrowing of the right and left pulmonary arteries (arrows). 


\section{ANSWER}

Perfusion scintigraphy demonstrated large bilateral perfusion defects, and the MR angiogram showed significant stenoses of the proximal portions of both pulmonary arteries (figure $1 \mathrm{~B}, \mathrm{D})$. The coronal CT image highlighted narrowing of the left pulmonary artery secondary to the large mass-like perihilar lesions of PMF (arrow, figure 1C). There was no evidence of large airway obstruction secondary to these lesions.

On right heart catheterisation there was evidence of pulmonary hypertension $(\mathrm{PH})$ with a mean pulmonary artery pressure of $39 \mathrm{~mm} \mathrm{Hg}$ and a moderate elevation of pulmonary vascular resistance (6 Wood units). The pulmonary capillary wedge pressure $(4 \mathrm{~mm} \mathrm{Hg})$, right atrial pressure $(3 \mathrm{~mm} \mathrm{Hg})$ and cardiac index $\left(3.26 \mathrm{~L} / \mathrm{min} / \mathrm{m}^{2}\right)$ were in the normal range. There was a wide pulmonary artery pulse pressure $(83 / 16 \mathrm{~mm} \mathrm{Hg})$, consistent with proximal rather than distal involvement of the pulmonary vasculature. A diagnosis of PH secondary to bilateral central pulmonary artery stenoses was made.

The patient did not require treatment for his $\mathrm{PH}$, but remained under review. He is now deceased from an unrelated condition.

\section{DISCUSSION}

PMF occurs when pulmonary nodules and hilar lymphadenopathy and calcification coalesce to form characteristic conglomerate masses. ${ }^{1} \mathrm{PH}$ is a recognised complication of PMF, but acquired bilateral pulmonary artery stenosis is rare, ${ }^{2}$ and we found no other reports of this in PMF. We hypothesise that extrinsic compression of the pulmonary vasculature by large conglomerate masses of fibrosis, typical features of PMF, caused the central vessel stenosis in our patient. Hilar and mediastinal lymphadenopathy can give a similar picture in sarcoidosis, ${ }^{3} 4$ and has been reported in a case of silicosis. ${ }^{5}$ Other causes of extrinsic pulmonary artery compression include aortic aneurysms, ${ }^{6}$ tumours, infection, cysts and conditions that cause mediastinal fibrosis. $^{7}$

In summary, we present a rare case of $\mathrm{PH}$ secondary to pulmonary artery stenosis in PMF. A CT chest to evaluate for cardiac, mediastinal, vascular and intrinsic lung disease, and a comprehensive assessment including cardiopulmonary MRI and perfusion scintigraphy, provide important information that supplements cardiac catheterisation in the assessment of suspected $\mathrm{PH}$.

Acknowledgements We would like to acknowledge Professor Jim Wild, University of Sheffield, for the development of the MRA imaging techniques used in this case.

Contributors ATG and JW article draft; KA article conception and critical revision; SR selection and interpretation of radiographic images; DGK critical revision.

Competing interests None declared.

Patient consent Obtained.

Provenance and peer review Not commissioned; externally peer reviewed.

\section{REFERENCES}

1 Narayanasamy S, Singh J, Anton $P$, et al. Angel's wing appearance on chest radiograph-progressive massive fibrosis. Clin Med 2015;15:307.

2 Viseur P, Unger P. Doppler echocardiographic diagnosis and follow-up of acquired pulmonary stenosis due to external cardiac compression. Cardiology 1995;86:80-2.

3 Damuth TE, Bower JS, Cho K, et al. Major pulmonary artery stenosis causing pulmonary hypertension in sarcoidosis. Chest 1980;78:888-91.

4 Nunes H, Humbert M, Capron F, et al. Pulmonary hypertension associated with sarcoidosis: mechanisms, haemodynamics and prognosis. Thorax 2006;61:68-74.

5 Mahnken AH, Breuer C, Haage P. Silicosis-induced pulmonary artery stenosis: demonstration by MR angiography and perfusion MRI. Br J Radiol 2001;74:859-61.

6 Okiwelu N, Finn C, Vanden Driesen R, et al. Ruptured penetrating ulcer of the ascending aorta with pulmonary artery compression. Asian Cardiovasc Thorac Ann 2016:24:266-8.

7 Kharwar RB, Sharma A, Sethi $R$, et al. Pulmonary artery stenosis due to lung carcinoma: a rare cause of dyspnea. J Cardiovasc Ultrasound 2014;22:209-12. 